\title{
Synthesis of Stable 2H-Pyran-5-carboxylates via a Catalyzed Propargyl-Claisen Rearrangement/Oxa-6 $\pi$ Electrocyclization Strategy
}

\author{
Helge Menz, Stefan F. Kirsch ${ }^{*}$ \\ Department Chemie, Technische Universität München, Lichtenbergstr. 4, D-85747 Garching, \\ Germany
}

\section{Supporting Information}

Representative experimental procedures for catalytic $2 \mathrm{H}$-pyran formation, compound characterization data for $\mathbf{3 a}-\mathbf{o}$, and copies of ${ }^{1} \mathrm{H}$ and ${ }^{13} \mathrm{C}$ NMR of $\mathbf{3}, \mathbf{5}-\mathbf{7}$.

(24 Pages)

\section{List of Contents}

$\mathrm{S} 1-\mathrm{S} 2$

S2

S2-S6

S7-S21

S22-S24
General experimental details

General procedure for the $2 \mathrm{H}$-pyran formation starting from propargyl vinyl ethers 1

Further characterization data for compounds $\mathbf{3 a}-\mathbf{o}$

${ }^{1} \mathrm{H}$ NMR and ${ }^{13} \mathrm{C}$ NMR spectra for compounds 3

${ }^{1} \mathrm{H}$ NMR and ${ }^{13} \mathrm{C}$ NMR spectra for compounds 5-7

General experimental details: All propargyl vinyl ethers 1 were synthesized by the reaction of the corresponding propargylic alcohols with 2-propynoic acid derivatives in the presence of trimethylphosphine or tributylphosphine. ${ }^{1}$ Ethyl 2-butynoate, methyl 2-octynoate, ethyl phenylpropiolate and 3-pentyn-2-ol were purchased from Aldrich. All commercially available chemicals were used without further purification. All the other propargylic alcohols were prepared from the corresponding alkynes by addition to the corresponding aldehyde. ${ }^{2} \mathrm{AgSbF}_{6}$ and $\mathrm{DBU}$ were purchased from Aldrich.

\footnotetext{
${ }^{1}$ Inanaga, J.; Baba, Y.; Hanamoto, T. Chem. Lett. 1993, 241-244.

${ }^{2}$ Hashmi, A. S. K.; Haufe, P.; Nass, A. R. Adv. Synth. Catal. 2003, 345, 1237-1241.
} 
${ }^{1} \mathrm{H}$ NMR spectra were obtained on Bruker $500 \mathrm{MHz}$ FT-NMR, $360 \mathrm{MHz}$ FT-NMR and $250 \mathrm{MHz}$ FTNMR spectrometers. ${ }^{13} \mathrm{C}$ NMR spectra were recorded at $90.6 \mathrm{MHz}$. Chemical shifts are reported in ppm relative to solvent signal. Multiplicity is indicated as follows: $\mathrm{s}$ (singlet); $\mathrm{d}$ (doublet); $\mathrm{t}$ (triplet); $\mathrm{q}$ (quartet); m (multiplet); dd (doublet of doublets); sep (septet). High resolution mass spectra and EI were determined on a Finnigan MAT 95S and MAT 8200. Flash chromatography was performed with E. Merck silica gel $(43-60 \mu \mathrm{m})$. The eluent used is reported in parentheses ( $\mathrm{P}=$ pentanes). Thin-layer chromatography (TLC) was performed on precoated glass-backed plates (Merck Kieselgel $60 \mathrm{~F}_{254}$ ), and components were visualized by observation under UV light or by treating the plates with $\mathrm{KMnO}_{4} / \mathrm{H}_{2} \mathrm{SO}_{4}$ followed by heating.

The $2 \mathrm{H}$-pyrans 3 show significant decomposition on silica gel during the purification process. Therefore, it is recommended to use a minimum amount of silica gel for flash chromatography. The use of neutral $\mathrm{Al}_{2} \mathrm{O}_{3}$ (Aldrich; Brockmann 1) did neither improve the yields nor the purity of the products.

\section{General Procedure for the 2H-Pyran Formation starting from Propargyl Vinyl Ethers 1.}

Ethyl 6-ethyl-2-methyl-4-phenyl-6H-pyran-3-carboxylate (3a)

$\mathrm{AgSbF}_{6}\left(38 \mathrm{mg}, 0.11 \mathrm{mmol}, 5 \mathrm{~mol} \%\right.$ ) was added to a solution of $\mathbf{1 a}(570 \mathrm{mg}, 2.1 \mathrm{mmol})$ in $\mathrm{CH}_{2} \mathrm{Cl}_{2}(10$ $\mathrm{ml}$ ), and the reaction vial was sealed, protected from light, and stirred at room temperature for $60 \mathrm{~min}$. Then, a solution of DBU (17 mg, $0.11 \mathrm{mmol}, 5 \mathrm{~mol} \%)$ in $\mathrm{CH}_{2} \mathrm{Cl}_{2}(0.5 \mathrm{~mL})$ was added, and the reaction mixture was stirred at room temperature for $30 \mathrm{~min}$ (until TLC analysis indicated complete conversion). The mixture was concentrated under reduced pressure. Purification of the residue by flash chromatography on silica gel $(\mathrm{P} / \mathrm{EtOAc}=80 / 20)$ gave $2 \mathrm{H}$-pyran 3a as a colorless oil (432 $\mathrm{mg}, 1.59$ mmol, 76\%). $\mathrm{R}_{\mathrm{f}}=0.65(\mathrm{P} / \mathrm{EtOAc}=80 / 20) ;{ }^{1} \mathrm{H} \mathrm{NMR}\left(360 \mathrm{MHz}, \mathrm{CDCl}_{3}\right) \delta 0.72(\mathrm{t}, J=7.1 \mathrm{~Hz}, 3 \mathrm{H})$, $1.04(\mathrm{t}, J=7.4 \mathrm{~Hz}, 3 \mathrm{H}), 1.74-1.96(\mathrm{~m}, 2 \mathrm{H}), 2.35$ (s, $3 \mathrm{H}), 3.81-3.87$ (m, $2 \mathrm{H}), 4.63$ (dt, $J=3.8,6.4$ $\mathrm{Hz}, 1 \mathrm{H}), 5.29$ (d, $J=3.8 \mathrm{~Hz}, 1 \mathrm{H}), 7.31-7.34(\mathrm{~m}, 3 \mathrm{H}), 7.44-7.48(\mathrm{~m}, 2 \mathrm{H}) ;{ }^{13} \mathrm{C}$ NMR $(90.6 \mathrm{MHz}$, $\left.\mathrm{CDCl}_{3}\right) \delta 9.6,13.8,19.0,27.3,60.0,77.9,108.3,116.3,126.8,127.3,128.3,137.4,141.1,166.0$, 167.6. LRMS (EI): $272(10 \%)\left[\mathrm{M}^{+}\right], 199$ (100\%); HRMS 272.1420 [272.1412 calcd for $\mathrm{C}_{17} \mathrm{H}_{20} \mathrm{O}_{3} \mathrm{Cl}$ $\left.\left(\mathrm{M}^{+}\right)\right]$. 


\section{Ethyl 4-(4-tert-butylphenyl)-6-ethyl-2-methyl-6H-pyran-3-carboxylate (3b)}

Following the general procedure, $\mathbf{3 b}$ was obtained as a colorless oil (77\%) after flash chromatography on silica gel $(\mathrm{P} / \mathrm{EtOAc}=80 / 20)$.

$\mathrm{R}_{\mathrm{f}}=0.78(\mathrm{P} / \mathrm{EA}=80 / 20) ;{ }^{1} \mathrm{H} \mathrm{NMR}\left(360 \mathrm{MHz}, \mathrm{CDCl}_{3}\right) \delta 0.65(\mathrm{t}, J=7.1 \mathrm{~Hz}, 3 \mathrm{H}), 1.02(\mathrm{t}, J=7.4 \mathrm{~Hz}$, 3 H), 1.30 (s, 9 H), 1.73-1.94 (m, 2 H), 2.33 (s, 3 H), 3.76-3.90 (m, 2 H), 4.61 (dt, J = 3.8, 6.4 Hz, 1 H), $5.28(\mathrm{~d}, J=3.8 \mathrm{~Hz}, 1 \mathrm{H}), 7.11(\mathrm{~d}, J=8.5 \mathrm{~Hz}, 2 \mathrm{H}), 7.29(\mathrm{~d}, J=8.5 \mathrm{~Hz}, 2 \mathrm{H}) ;{ }^{13} \mathrm{C}$ NMR $(90.6$ $\left.\mathrm{MHz}, \mathrm{CDCl}_{3}\right) \delta$ 9.6, 13.5, 18.9, 27.3, 31.7, 34.8, 60.0, 77.9, 108.6, 115.7, 125.2, 126.5, 137.1, 138.1, 150.4, 165.8, 167.8. LRMS (EI): 328 (4\%) [M+], 271 (48\%), 161 (100\%); HRMS 328.2030 [328.2038 calcd for $\left.\mathrm{C}_{21} \mathrm{H}_{28} \mathrm{O}_{3}\left(\mathrm{M}^{+}\right)\right]$.

\section{Ethyl 6-ethyl-2-methyl-4-(4-phenoxyphenyl)-6H-pyran-3-carboxylate (3c)}

Following the general procedure, 3c was obtained as a colorless solid (63\%) after flash chromatography on silica gel $(\mathrm{P} / \mathrm{EtOAc}=80 / 20)$.

$\mathrm{R}_{\mathrm{f}}=0.59(\mathrm{P} / \mathrm{EA}=95 / 5) ;{ }^{1} \mathrm{H} \mathrm{NMR}\left(360 \mathrm{MHz}, \mathrm{CDCl}_{3}\right) \delta 0.85(\mathrm{t}, J=7.2 \mathrm{~Hz}, 3 \mathrm{H}), 1.04(\mathrm{t}, J=7.4 \mathrm{~Hz}, 3$ H), 1.74-1.96 (m, 2 H), 2.34 (s, 3 H), 3.87-3.94 (m, 2 H), 4.61 (dt, J = 3.6, 6.6 Hz, 1 H), 5.28 (d, J = $3.6 \mathrm{~Hz}, 1 \mathrm{H}), 6.93$ (d, $J=8.6 \mathrm{~Hz}, 2 \mathrm{H}), 6.99$ (d, $J=7.5 \mathrm{~Hz}, 2 \mathrm{H}), 7.09$ (t. $J=7.5 \mathrm{~Hz}, 1 \mathrm{H}), 7.17$ (d, $J=$ $8.6 \mathrm{~Hz}, 2 \mathrm{H}), 7.32(\mathrm{t}, J=7.5 \mathrm{~Hz}, 2 \mathrm{H}) ;{ }^{13} \mathrm{C} \mathrm{NMR}\left(90.6 \mathrm{MHz}, \mathrm{CDCl}_{3}\right) \delta$ 9.6, 14.0, 19.1, 27.2, 60.1, $77.9,108.5,116.0,188.9,119.0,123.5,128.2,130.1,136.3,136.7,156.7,157.7,166.1,167.6$. LRMS (EI): $364(8 \%)\left[\mathrm{M}^{+}\right], 291$ (100\%), 235 (95\%), 198 (48\%), 142 (68\%); HRMS 364.1674 [364.1675 calcd for $\left.\mathrm{C}_{23} \mathrm{H}_{24} \mathrm{O}_{4}\left(\mathrm{M}^{+}\right)\right]$.

\section{Ethyl 6-ethyl-2-methyl-4-(thiophen-3-yl)-6H-pyran-3-carboxylate (3d)}

Following the general procedure, 3d was obtained as a colorless solid (90\%) after flash chromatography on silica gel $(\mathrm{P} /$ EtOAc $=80 / 20)$.

$\mathrm{R}_{\mathrm{f}}=0.54(\mathrm{P} / \mathrm{EA}=80 / 20) ;{ }^{1} \mathrm{H} \mathrm{NMR}\left(360 \mathrm{MHz}, \mathrm{CDCl}_{3}\right) \delta 0.88(\mathrm{t}, J=7.1 \mathrm{~Hz}, 3 \mathrm{H}), 1.02(\mathrm{t}, J=7.4 \mathrm{~Hz}$, $3 \mathrm{H}), 1.73-1.91$ (m, $2 \mathrm{H}), 2.30$ (s, $3 \mathrm{H}), 3.93(\mathrm{dq}, J=1.9,7.1 \mathrm{~Hz}, 2 \mathrm{H}), 4.58(\mathrm{dt}, J=3.7,6.8 \mathrm{~Hz}, 1 \mathrm{H})$, $5.35(\mathrm{~d}, J=3.6 \mathrm{~Hz}, 1 \mathrm{H}), 6.93(\mathrm{dd}, J=1.4,5.0 \mathrm{~Hz}, 1 \mathrm{H}), 7.07$ (dd, $J=1.4,3.0 \mathrm{~Hz}, 1 \mathrm{H}), 7.21$ (dd, $J=$ 3.0, $5.0 \mathrm{~Hz}, 1 \mathrm{H}) ;{ }^{13} \mathrm{C} \mathrm{NMR}\left(90.6 \mathrm{MHz}, \mathrm{CDCl}_{3}\right) \delta 9.6,13.9,19.0,27.2,60.2,77.7,108.6,115.7$, 120.75, 125.2, 127.2, 132.1, 141.5, 165.4, 167.7. LRMS (EI): 278 (5\%) $\left[\mathrm{M}^{+}\right], 205(82 \%), 149$ (100\%), 134 (60\%); HRMS 278.0971 [278.0977 calcd for $\left.\mathrm{C}_{15} \mathrm{H}_{18} \mathrm{O}_{3} \mathrm{~S}\left(\mathrm{M}^{+}\right)\right]$.

\section{Ethyl 4-[2-(tert-butyldimethylsilanyloxy)ethyl]-6-ethyl-2-methyl-6H-pyran-3-carboxylate} (3e)

Following the general procedure, $\mathbf{3 e}$ was obtained as a colorless oil (82\%) after flash chromatography on silica gel $(\mathrm{P} / \mathrm{EtOAc}=80 / 20)$.

$\mathrm{R}_{\mathrm{f}}=0.79(\mathrm{P} / \mathrm{EA}=80 / 20) ;{ }^{1} \mathrm{H} \mathrm{NMR}\left(360 \mathrm{MHz}, \mathrm{CDCl}_{3}\right) \delta 0.01(\mathrm{~s}, 6 \mathrm{H}), 0.87(\mathrm{~s}, 9 \mathrm{H}), 0.97(\mathrm{t}, J=7.1$ $\mathrm{Hz}, 3 \mathrm{H}), 1.31$ (t, $J=7.4 \mathrm{~Hz}, 3 \mathrm{H}), 1.63-1.80$ (m, $2 \mathrm{H}), 2.17$ (s, $3 \mathrm{H}), 2.46-2.61$ (m, $2 \mathrm{H}), 3.61$ (dt, $J=$ 
$1.2,6.8 \mathrm{~Hz}, 2 \mathrm{H}), 4.21$ (q, $J=7.1 \mathrm{~Hz}, 2 \mathrm{H}), 4.41-4.46(\mathrm{~m}, 1 \mathrm{H}), 5.09$ (d, $J=3.4 \mathrm{~Hz}, 1 \mathrm{H}) ;{ }^{13} \mathrm{C}$ NMR $\left(90.6 \mathrm{MHz}, \mathrm{CDCl}_{3}\right) \delta-5.0,9.5,14.6,18.7,19.9,26.3,27.5,37.1,60.3,62.9,77.5,108.3,116.1,132.3$, 164.6, 167.7. LRMS (EI): 354 (2\%) [M+], 297 (42\%), 89 (100\%); HRMS 354.2217 [354.2226 calcd for $\left.\mathrm{C}_{19} \mathrm{H}_{34} \mathrm{O}_{4} \mathrm{Si}\left(\mathrm{M}^{+}\right)\right]$.

\section{Ethyl 4-(cyclohexylmethyl)-6-ethyl-2-methyl-6H-pyran-3-carboxylate (3f)}

Following the general procedure, $\mathbf{3 f}$ was obtained as a colorless oil (75\%) after flash chromatography on silica gel $(\mathrm{P} / \mathrm{EtOAc}=80 / 20)$.

$\mathrm{R}_{\mathrm{f}}=0.58(\mathrm{P} / \mathrm{EA}=95 / 5) ;{ }^{1} \mathrm{H}$ NMR $\left(360 \mathrm{MHz}, \mathrm{CDCl}_{3}\right) \delta 0.96(\mathrm{t}, J=7.5 \mathrm{~Hz}, 3 \mathrm{H}), 1.11-1.21(\mathrm{~m}, 5 \mathrm{H})$, $1.31(\mathrm{t}, J=7.2 \mathrm{~Hz}, 3 \mathrm{H}), 1.62-1.80(\mathrm{~m}, 8 \mathrm{H}), 2.05-2.11(\mathrm{~m}, 1 \mathrm{H}), 2.17(\mathrm{~s}, 3 \mathrm{H}), 2.26-2.30(\mathrm{~m}, 1 \mathrm{H})$, $4.21(\mathrm{dq}, J=2.4,7.1 \mathrm{~Hz}, 2 \mathrm{H}), 4.43-4.48(\mathrm{~m}, 1 \mathrm{H}), 4.97(\mathrm{~d}, J=3.4 \mathrm{~Hz}, 1 \mathrm{H}) ;{ }^{13} \mathrm{C}$ NMR $(90.6 \mathrm{MHz}$, $\left.\mathrm{CDCl}_{3}\right) \delta 9.5,14.7,19.7,26.6,26.7,27.0,27.6,33.2,33.8,36.7,41.9,60.3,77.5,108.3,115.6,133.6$, 164.1, 168.1. LRMS (EI): 292 (7\%) [M+], 249 (100\%), 219 (69\%); HRMS 292.2041 [292.2038 calcd for $\mathrm{C}_{18} \mathrm{H}_{28} \mathrm{O}_{3}\left(\mathrm{M}^{+}\right)$].

\section{Ethyl 2,6-dimethyl-4-phenyl-6H-pyran-3-carboxylate (3g)}

Following the general procedure, $\mathbf{3 g}$ was obtained as a colorless solid (72\%) after flash chromatography on silica gel $(\mathrm{P} /$ EtOAc $=80 / 20)$.

$\mathrm{R}_{\mathrm{f}}=0.56(\mathrm{P} / \mathrm{EA}=95 / 5) ;{ }^{1} \mathrm{H}$ NMR $\left(360 \mathrm{MHz}, \mathrm{CDCl}_{3}\right) \delta 0.72(\mathrm{t}, J=7.1 \mathrm{~Hz}, 3 \mathrm{H}), 1.49(\mathrm{~d}, J=6.6 \mathrm{~Hz}, 3$ H), 2.34 (s, 3 H), 3.80-3.87 (m, 2 H), 4.81 (dq, $J=3.8,6.6$ Hz, 1 H), 5.26 (d, $J=3.5$ Hz, 1 H), $7.18-$ 7.20 (m, $2 \mathrm{H}), 7.24-7.30(\mathrm{~m}, 3 \mathrm{H}) ;{ }^{13} \mathrm{C}$ NMR $\left(90.6 \mathrm{MHz}, \mathrm{CDCl}_{3}\right) \delta$ 13.7, 19.0, 20.2, 60.0, 73.0, 108.5, 117.5, 126.8, 127.3, 128.3, 137.2, 141.0, 166.0, 167.5. LRMS (EI): 258 (32\%) [M ], 243 (100\%), 215 (22\%); HRMS $258.1251\left[258.1256\right.$ calcd for $\left.\mathrm{C}_{16} \mathrm{H}_{18} \mathrm{O}_{3}\left(\mathrm{M}^{+}\right)\right]$.

\section{Ethyl 6-iso-propyl-2-methyl-4-phenyl-6H-pyran-3-carboxylate (3h)}

Following the general procedure, $\mathbf{3 h}$ was obtained as a colorless solid (80\%) after flash chromatography on silica gel $(\mathrm{P} /$ EtOAc $=80 / 20)$.

$\mathrm{R}_{\mathrm{f}}=0.54(\mathrm{P} / \mathrm{EA}=95 / 5) ;{ }^{1} \mathrm{H} \mathrm{NMR}\left(250 \mathrm{MHz}, \mathrm{CDCl}_{3}\right) \delta 0.71(\mathrm{t}, J=7.1 \mathrm{~Hz}, 3 \mathrm{H}), 1.01(\mathrm{~d}, J=6.6 \mathrm{~Hz}, 3$ H), 1.03 (d, $J=6.8 \mathrm{~Hz}, 3 \mathrm{H}), 2.05-2.19$ (m, $1 \mathrm{H}), 2.35$ (s, $3 \mathrm{H}), 3.83$ (q, $J=7.1 \mathrm{~Hz}, 2 \mathrm{H}), 4.40$ (dd, $J=$ 3.9, $6.4 \mathrm{~Hz}, 1 \mathrm{H}), 5.33$ (d, $J=3.8 \mathrm{~Hz}, 1 \mathrm{H}), 7.18-7.21$ (m, $2 \mathrm{H}), 7.23-7.29$ (m, $3 \mathrm{H}) ;{ }^{13} \mathrm{C}$ NMR $(62.9$ $\left.\mathrm{MHz}, \mathrm{CDCl}_{3}\right) \delta 13.7,18.3,18.3,19.0,31.8,60.0,81.6,108.4,115.0,126.8,127.3,128.3,137.5$, 141.3, 166.1, 167.7.

\section{Ethyl 6-benzyl-2-methyl-4-phenyl-6H-pyran-3-carboxylate (3i)}

Following the general procedure, 3i was obtained as a colorless solid $(50 \%)$ after flash chromatography on silica gel $(\mathrm{P} / \mathrm{EtOAc}=80 / 20)$. 
$\mathrm{R}_{\mathrm{f}}=0.50(\mathrm{P} / \mathrm{EA}=95 / 5) ;{ }^{1} \mathrm{H} \mathrm{NMR}\left(360 \mathrm{MHz}, \mathrm{CDCl}_{3}\right) \delta 0.73(\mathrm{t}, J=7.2 \mathrm{~Hz}, 3 \mathrm{H}), 2.34(\mathrm{~s}, 3 \mathrm{H}), 3.03$ (dd, $J=6.8,13.6 \mathrm{~Hz}, 1 \mathrm{H}), 3.21(\mathrm{dd}, J=7.3,13.6 \mathrm{~Hz}, 1 \mathrm{H}), 3.85$ (q, $J=7.2 \mathrm{~Hz}, 2 \mathrm{H}), 4.95$ (dt, $J=4.1$, $7.0 \mathrm{~Hz}, 1 \mathrm{H}), 5.32$ (d, J=4.1 Hz, $1 \mathrm{H}), 7.17-7.20$ (m, $2 \mathrm{H}), 7.26-7.31$ (m, $6 \mathrm{H}), 7.33-7.38$ (m, $2 \mathrm{H})$; ${ }^{13} \mathrm{C}$ NMR $\left(90.6 \mathrm{MHz}, \mathrm{CDCl}_{3}\right) \delta 13.7,19.1,40.4,60.1,77.1,108.7,115.8,126.8,127.0,127.4,128.3$,

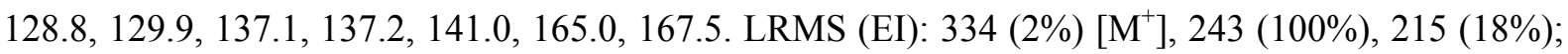
HRMS 334.1559 [334.1569 calcd for $\mathrm{C}_{22} \mathrm{H}_{22} \mathrm{O}_{3}\left(\mathrm{M}^{+}\right)$].

\section{Ethyl 2-methyl-4-phenyl-6H-pyran-3-carboxylate (3j)}

Following the general procedure, $\mathbf{3 j}$ was obtained as a colorless solid (61\%) after flash chromatography on silica gel $(\mathrm{P} / \mathrm{EtOAc}=80 / 20)$.

$\mathrm{R}_{\mathrm{f}}=0.45(\mathrm{P} / \mathrm{EA}=95 / 5) ;{ }^{1} \mathrm{H}$ NMR $\left(360 \mathrm{MHz}, \mathrm{CDCl}_{3}\right) \delta 0.74(\mathrm{t}, J=7.1 \mathrm{~Hz}, 3 \mathrm{H}), 2.37(\mathrm{~s}, 3 \mathrm{H}), 3.86$ (q, $J=7.1 \mathrm{~Hz}, 2 \mathrm{H}), 4.67$ (d, $J=4.6 \mathrm{~Hz}, 2 \mathrm{H}), 5.45$ (t, $J=4.6 \mathrm{~Hz}, 1 \mathrm{H}), 7.19-7.22$ (m, $2 \mathrm{H}), 7.26-7.30$ $(\mathrm{m}, 3 \mathrm{H}) ;{ }^{13} \mathrm{C}$ NMR $\left(90.6 \mathrm{MHz}, \mathrm{CDCl}_{3}\right) \delta 13.7,18.7,60.1,66.1,109.2,111.7,126.8,127.4,128.4$, 137.9, 140.8, 166.9, 167.4. LRMS (EI): $244(5 \%)\left[\mathrm{M}^{+}\right], 201$ (9\%), 171 (28\%), 115 (100\%); HRMS 244.1097 [244.1099 calcd for $\left.\mathrm{C}_{15} \mathrm{H}_{16} \mathrm{O}_{3}\left(\mathrm{M}^{+}\right)\right]$.

\section{Ethyl 4-(2-methoxyphenyl)-2-methyl-6H-pyran-3-carboxylate (3k)}

Following the general procedure, 3k was obtained as a colorless solid (59\%) after flash chromatography on silica gel $(\mathrm{P} /$ EtOAc $=80 / 20)$.

$\mathrm{R}_{\mathrm{f}}=0.49(\mathrm{P} / \mathrm{EA}=95 / 5) ;{ }^{1} \mathrm{H}$ NMR $\left(250 \mathrm{MHz}, \mathrm{CDCl}_{3}\right) \delta 0.75(\mathrm{t}, J=7.1 \mathrm{~Hz}, 3 \mathrm{H}), 2.33(\mathrm{~s}, 3 \mathrm{H}), 3.73(\mathrm{~s}$, $3 \mathrm{H}), 3.82$ (q, $J=7.1 \mathrm{~Hz}, 2 \mathrm{H}), 4.68$ (d, $J=4.3 \mathrm{~Hz}, 2 \mathrm{H}), 5.42$ (t, $J=4.3 \mathrm{~Hz}, 1 \mathrm{H}), 6.80$ (d, $J=8.0 \mathrm{~Hz}$, $1 \mathrm{H}), 6.92(\mathrm{t}, J=7.4 \mathrm{~Hz}, 1 \mathrm{H}), 7.13-7.27(\mathrm{~m}, 2 \mathrm{H}) ;{ }^{13} \mathrm{C} \mathrm{NMR}\left(62.9 \mathrm{MHz}, \mathrm{CDCl}_{3}\right) \delta 13.7,19.2,55.7$, 59.7, 65.8, 109.6, 110.3, 120.9, 128.7, 129.6, 130.4, 134.8, 156.7, 164.8, 167.2. LRMS (EI): 274 $(10 \%)\left[\mathrm{M}^{+}\right], 201(82 \%), 145(100 \%)$; HRMS 274.1197 [274.1205 calcd for $\mathrm{C}_{16} \mathrm{H}_{18} \mathrm{O}_{4}\left(\mathrm{M}^{+}\right)$].

\section{Ethyl 2-methyl-4-(thiophen-3-yl)-6H-pyran-3-carboxylate (3I)}

Following the general procedure, 3I was obtained as a colorless solid (53\%) after flash chromatography on silica gel $(\mathrm{P} /$ EtOAc $=80 / 20)$.

$\mathrm{R}_{\mathrm{f}}=0.47(\mathrm{P} / \mathrm{EA}=95 / 5) ;{ }^{1} \mathrm{H}$ NMR $\left(360 \mathrm{MHz}, \mathrm{CDCl}_{3}\right) \delta 0.88(\mathrm{t}, J=7.1 \mathrm{~Hz}, 3 \mathrm{H}), 2.31(\mathrm{~s}, 3 \mathrm{H}), 3.93$ (q, $J=7.1 \mathrm{~Hz}, 2 \mathrm{H}), 4.61(\mathrm{~d}, J=4.6 \mathrm{~Hz}, 2 \mathrm{H}), 5.49$ (t, $J=4.6 \mathrm{~Hz}, 1 \mathrm{H}), 6.94(\mathrm{dd}, J=1.4,5.0 \mathrm{~Hz}, 1 \mathrm{H})$, $7.09(\mathrm{dd}, J=1.4,3.0 \mathrm{~Hz}, 1 \mathrm{H}), 7.24(\mathrm{dd}, J=3.0,5.0 \mathrm{~Hz}, 1 \mathrm{H}) ;{ }^{13} \mathrm{C} \mathrm{NMR}\left(90.6 \mathrm{MHz}, \mathrm{CDCl}_{3}\right) \delta 13.9$, 18.7, 60.3, 65.8, 109.3, 111.1, 120.9, 125.3, 127.1, 132.6, 141.2, 166.2, 167.5. LRMS (EI): 250 (6\%) $\left[\mathrm{M}^{+}\right], 177$ (44\%), $121(100 \%)$; HRMS 250.0657 [250.0664 calcd for $\left.\mathrm{C}_{13} \mathrm{H}_{14} \mathrm{O}_{3} \mathrm{~S}\left(\mathrm{M}^{+}\right)\right]$.

\section{Methyl 6-ethyl-2-pentyl-4-phenyl-6H-pyran-3-carboxylate (3m)}

Following the general procedure, 3m was obtained as a colorless solid (72\%) after flash chromatography on silica gel $(\mathrm{P} /$ EtOAc $=80 / 20)$. 
$\mathrm{R}_{\mathrm{f}}=0.60(\mathrm{P} / \mathrm{EA}=95 / 5) ;{ }^{1} \mathrm{H} \mathrm{NMR}\left(360 \mathrm{MHz}, \mathrm{CDCl}_{3}\right) \delta 0.91(\mathrm{t}, J=7.1 \mathrm{~Hz}, 3 \mathrm{H}), 1.04(\mathrm{t}, J=7.4 \mathrm{~Hz}, 3$ H), 1.35-1.37 (m, $4 \mathrm{H}), 1.62-1.64(\mathrm{~m}, 2 \mathrm{H}), 1.77-1.91(\mathrm{~m}, 2 \mathrm{H}), 2.60-2.68(\mathrm{~m}, 1 \mathrm{H}), 2.77-2.86(\mathrm{~m}$, 1H), 3.34 (s, $3 \mathrm{H}), 4.58(\mathrm{dq}, J=3.8,6.8 \mathrm{~Hz}, 1 \mathrm{H}), 5.31$ (d, $J=3.8 \mathrm{~Hz}, 1 \mathrm{H}), 7.18-7.20$ (m, $2 \mathrm{H}), 7.24-$ 7.30 (m, $3 \mathrm{H}) ;{ }^{13} \mathrm{C}$ NMR $\left(90.6 \mathrm{MHz}, \mathrm{CDCl}_{3}\right) \delta$ 9.7, 14.3, 22.8, 27.3, 27.9, 31.9, 32.1, 51.1, 77.9, 108.2, 116.4, 126.6, 127.4, 128.4, 137.2, 140.8, 168.0, 169.3. LRMS (EI): 314 (5\%) [M $\left.{ }^{+}\right], 299$ (32\%), 103 (100\%); HRMS 314.1878 [314.1882 calcd for $\left.\mathrm{C}_{20} \mathrm{H}_{26} \mathrm{O}_{3}\left(\mathrm{M}^{+}\right)\right]$.

\section{Ethyl 6-ethyl-2,4-diphenyl-6H-pyran-3-carboxylate (3n) / 3n:trans-4n:cis-4n = 20:30:50}

Following the general procedure, the product mixture was obtained as a colorless oil (90\%) after flash chromatography on silica gel $(\mathrm{P} /$ EtOAc $=80 / 20)$. The ratio $3 \mathbf{n}$ :trans-4n:cis-4n $=20: 30: 50$ was determined by ${ }^{1} \mathrm{H}$ NMR of the purified compound.

${ }^{1} \mathrm{H}$ NMR $\left(360 \mathrm{MHz}, \mathrm{CDCl}_{3}\right) \delta$ 0.75-0.86 (m, 3n+trans-4n+cis-4n), 1.09-1.13 (m, 3n+trans-4n+cis4n), 1.59-1.67 (m, 3n+trans-4n+cis-4n), 1.91-2.03 (m, 3n+trans-4n+cis-4n), 3.73-3.80 (m, $0.4 \mathrm{H}$, 3n), 3.87 (q, $J=7.1 \mathrm{~Hz}, 0.6 \mathrm{H}$, trans-4n), 4.09-4.21 (m, $1 \mathrm{H}$, cis-4n), 4.80-4.85 (m, 0.2 H, 3n), 5.41 (d, $J=3.9 \mathrm{~Hz}, 0.2 \mathrm{H}, 3 \mathbf{n}), 5.63-5.73$ (m, $0.3 \mathrm{H}$, trans-4n), 5.86 (td, $J=7.5,11.8 \mathrm{~Hz}, 0.5 \mathrm{H}$, cis-4n), $6.31(\mathrm{~d}, J=15.2 \mathrm{~Hz}, 0.3 \mathrm{H}$, trans-4n), 6.77 (td, $J=1.6,11.7 \mathrm{~Hz}, 0.5 \mathrm{H}$, cis-4n), 7.09-7.16 (m, 3n+trans-4n+cis-4n), 7.25-7.33 (m, 3n+trans-4n+cis-4n), 7.40-7.51 (m, 3n+trans-4n+cis-4n), 7.577.65 (m, 3n+trans-4n+cis-4n), 7.75-7.77 (m, 3n+trans-4n+cis-4n), 7.99-8.02 (m, A+B+C); ${ }^{13} \mathrm{C} \mathrm{NMR}$ (the signals are not assigned to structures; $90 \mathrm{MHz}, \mathrm{CDCl}_{3}$ ) $\delta 9.8,13.0,13.6,13.8,13.9,14.2,14.5$, $23.1,26.7,27.4,61.3,127.4,127.5,127.6,128.0,128.2,128.3,128.5,128.6,128.9,128.9,129.0$, 129.1, 129.2, 129.3, 129.4, 129.5, 129.9, 1302, 131.7, 133.1, 133.8, 137.8, 138.0, 139.5, 142.1, 147.8, 152.6, 153.1, 165.4, 165.5, 195.0, 195.0.

\section{Ethyl 2-acetyl-3-cyclohexylmethyl-5-phenylpenta-2,4-diencarboxylate (40) / 3o:trans-} 4o:cis-4o $=0: 100: 0$

Following the general procedure, 40 was obtained as a colorless oil (36\%) after flash chromatography on silica gel $(\mathrm{P} / \mathrm{EtOAc}=80 / 20)$.

$\mathrm{R}_{\mathrm{f}}=0.41(\mathrm{P} / \mathrm{EA}=95 / 5) ;{ }^{1} \mathrm{H}$ NMR $(360 \mathrm{MHz}, \mathrm{CDCl} 3) \delta 0.98-1.07(\mathrm{~m}, 2 \mathrm{H}), 1.11-1.21(\mathrm{~m}, 3 \mathrm{H}), 1.32$ (t, $J=7.1 \mathrm{~Hz}, 3 \mathrm{H}), 1.57-1.76$ (m, $6 \mathrm{H}), 2.37$ (s, $3 \mathrm{H}), 2.73$ (d, $J=7.0 \mathrm{~Hz}, 2 \mathrm{H}), 4.27$ (q, $J=7.1 \mathrm{~Hz}, 2$ H), $6.99(\mathrm{~d}, J=16.1 \mathrm{~Hz}, 1 \mathrm{H}), 7.14(\mathrm{~d}, J=16.1 \mathrm{~Hz}, 1 \mathrm{H}), 7.30-7.37(\mathrm{~m}, 3 \mathrm{H}), 7.45-7.48(\mathrm{~m}, 2 \mathrm{H}) ;{ }^{13} \mathrm{C}$ NMR (90.6 MHz, CDCl3) $\delta 14.5,26.7,26.8,31.7,33.8,36.5,39.1,61.3,126.7,127.8,129.1,129.4$, 133.8, 136.7, 137.3, 151.2, 166.3, 201.4. LRMS (EI): 340 (11\%) [M+], 243 (100\%), 168 (40\%), 105 (48\%); HRMS 340.2037 [340.2039 calcd for $\left.\mathrm{C}_{22} \mathrm{H}_{28} \mathrm{O}_{3}\left(\mathrm{M}^{+}\right)\right]$. 

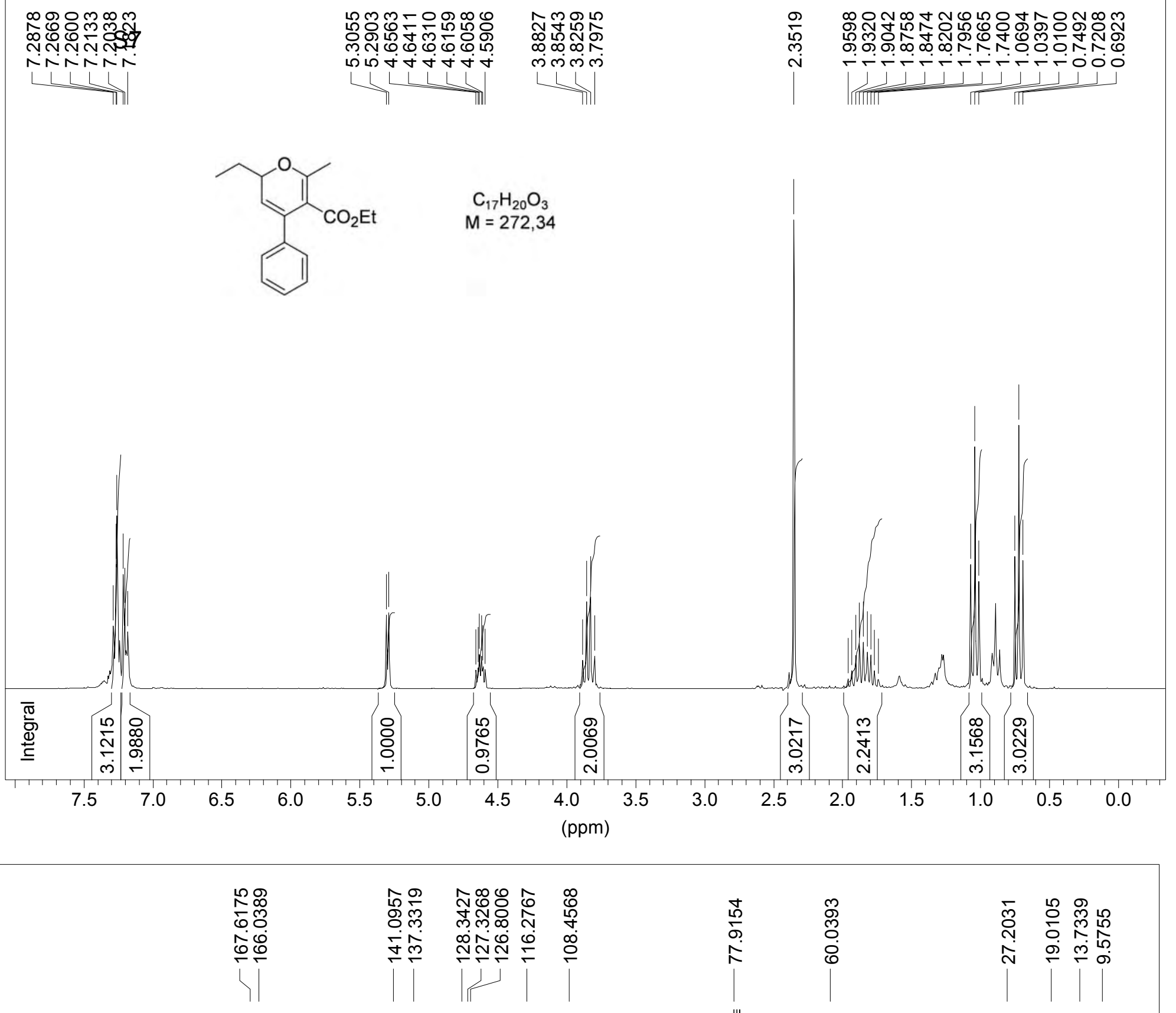

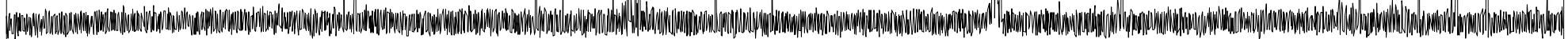



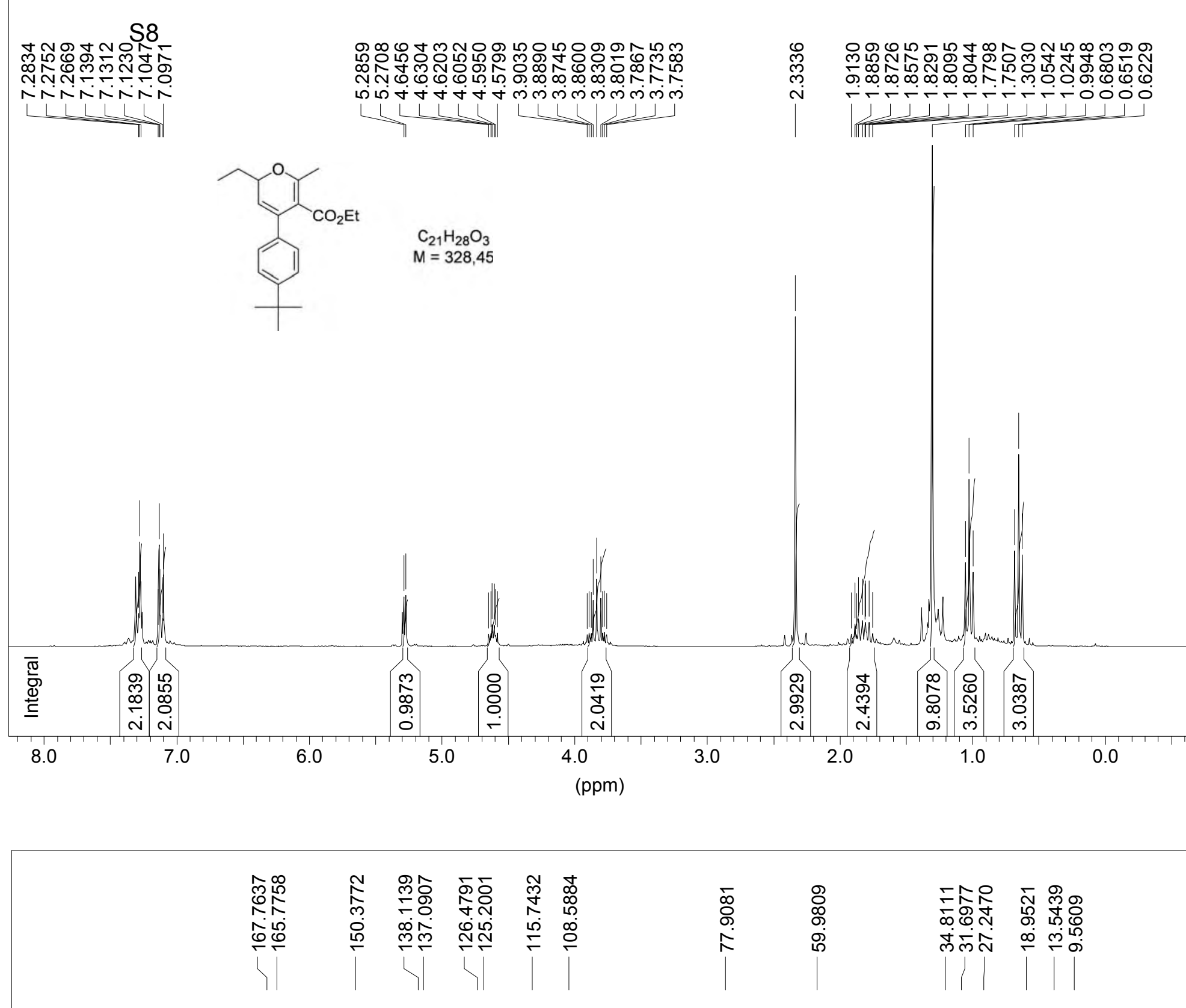

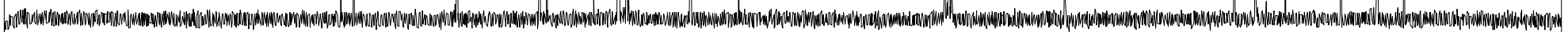




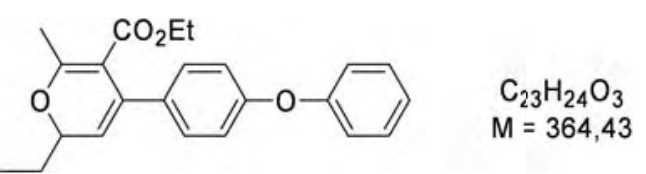

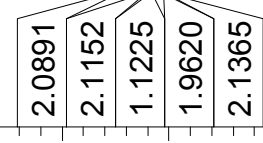

7.0

$\mathrm{C}_{23} \mathrm{H}_{24} \mathrm{O}_{3}$

$\mathrm{M}=364,43$

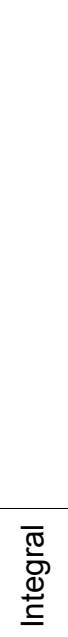

8.0

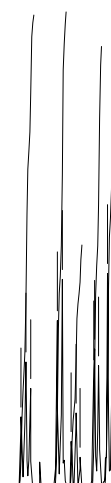

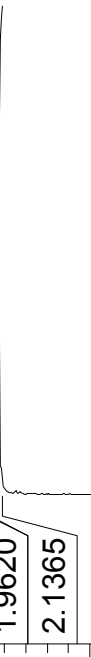

6.0
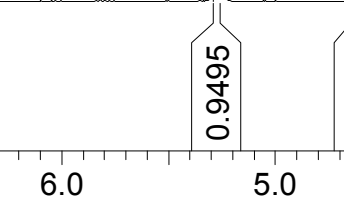

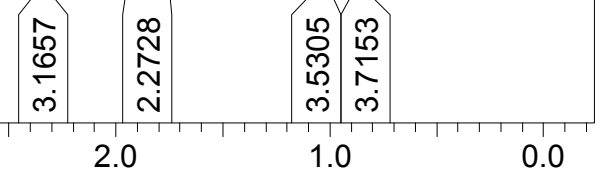

1.0

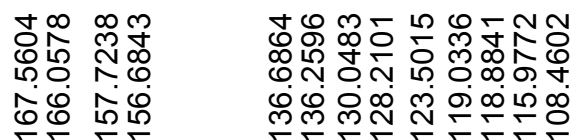




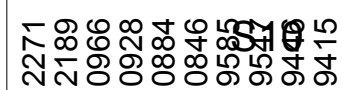

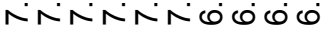

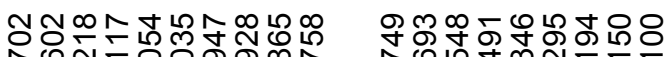

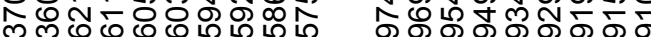
ம்<smiles>CCOC(=O)C1=C(C)OC(CC)C=C1c1ccsc1</smiles>

$M=278,37$
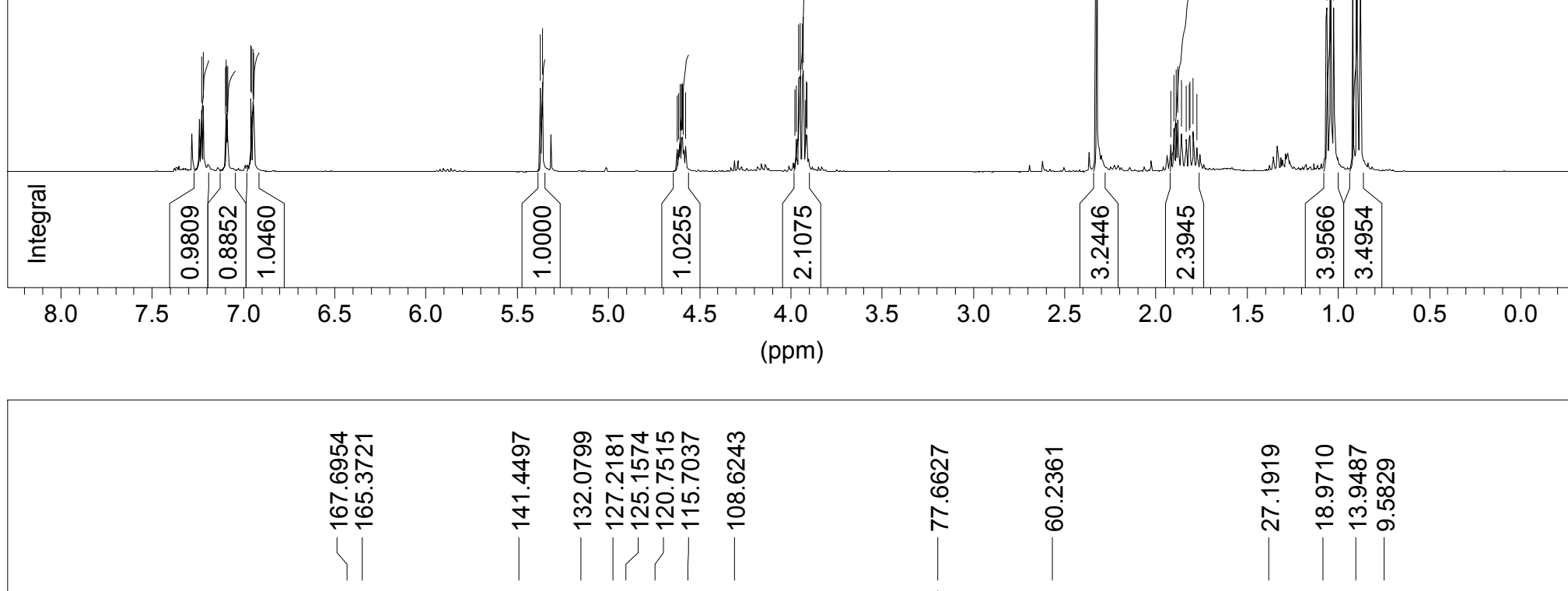
<smiles>CCOC(=O)C1=C(C)OC(CC)C=C1CC[OH+]S(C)(=O)=O</smiles>

OTBDMS

\begin{tabular}{|c|c|c|c|c|c|c|c|c|c|c|c|c|c|c|c|c|}
\hline & & & & & $\underset{\text { ৪ }}{\stackrel{8}{\circ}}$ & \& & 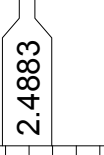 & $\begin{array}{l}\text { Õ } \\
\stackrel{0}{0} \\
\text { Ni }\end{array}$ & & 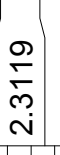 & $\begin{array}{l}\stackrel{2}{O} \\
\stackrel{\sim}{+} \\
\end{array}$ & 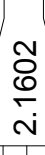 & $\begin{array}{l}\rho \\
\hat{N} \\
0 \\
0\end{array}$ & & $\begin{array}{l}\infty \\
0 \\
\text { m. } \\
\stackrel{0}{0}\end{array}$ & \\
\hline 7.5 & 7.0 & 6.5 & 6.0 & 5.5 & 5.0 & 4.5 & 4.0 & 3.5 & 3.0 & 2.5 & & & & 1.0 & & 0.5 \\
\hline
\end{tabular}

พิำ

응

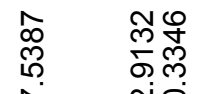

र

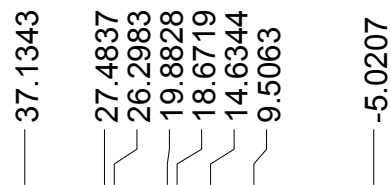




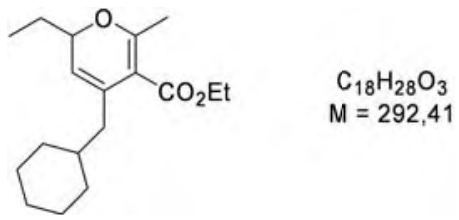

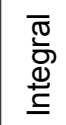

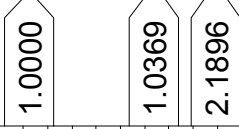

$7.5 \quad 7.0$

6.5

6.0

5.0

4.5

4.0

(ppm)

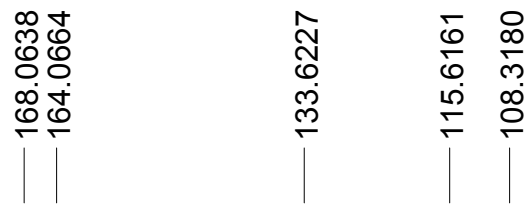

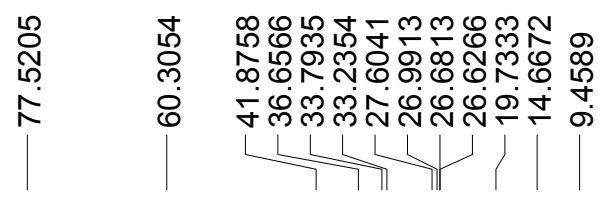

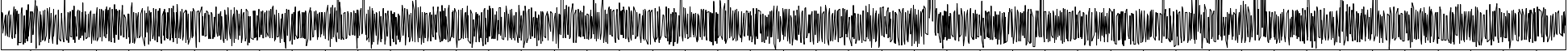



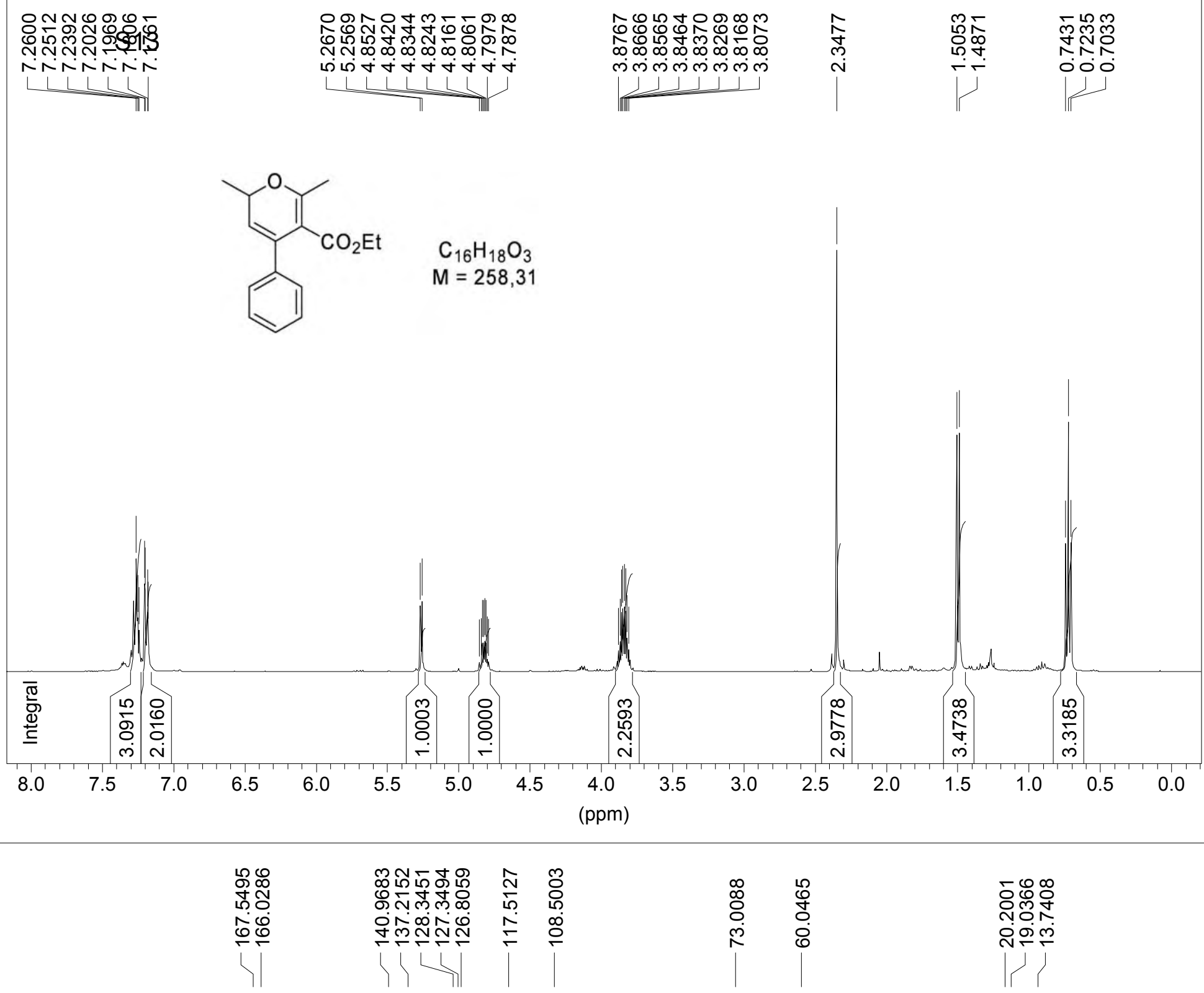

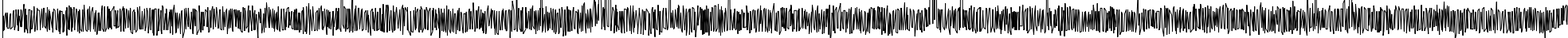




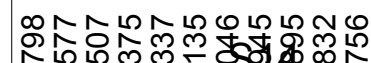

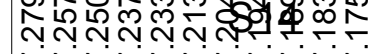

NNNNNNNN
항요

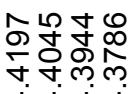

(م)

$\lfloor\leq$

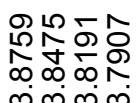

ल लm

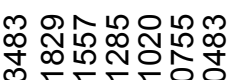

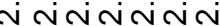

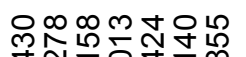

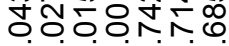

$\mathrm{C}_{18} \mathrm{H}_{22} \mathrm{O}_{3}$

$M=286,37$<smiles>c1ccccc1</smiles>

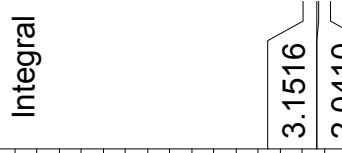

8.0

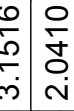

లి

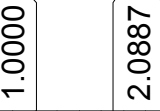

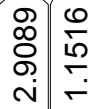

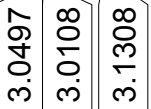

$\begin{array}{ll}7.0 & 6.0\end{array}$

5.0

4.0

3.0

2.0

1.0

0.0

(ppm)

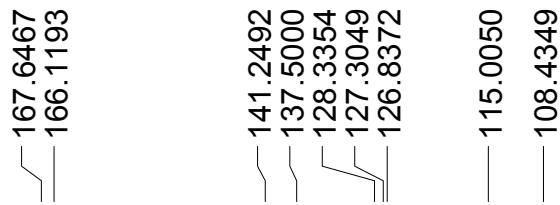

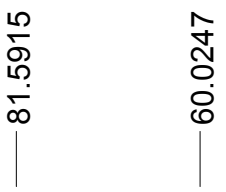

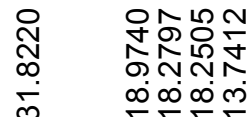


<smiles>CCOC(=O)C1=C(C)OC(Cc2ccccc2)C=C1c1ccccc1</smiles><smiles>CCCO</smiles>

(ppm)

3.0

2.0

0
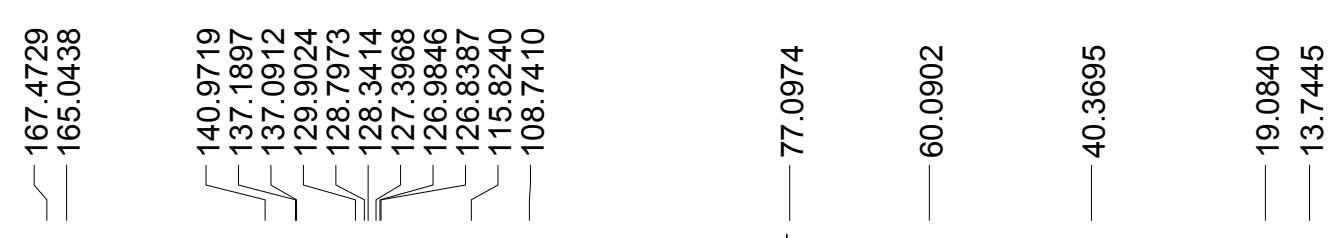

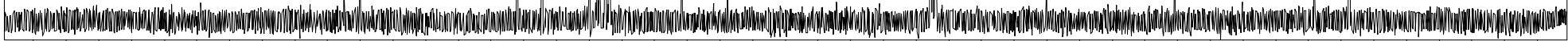




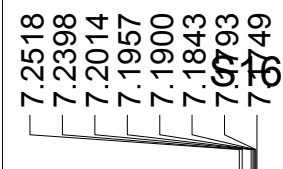

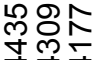

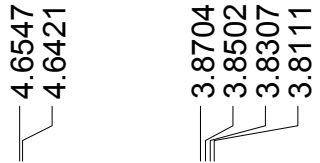<smiles>CCOC(=O)C1=C(C)OCC=C1c1ccccc1</smiles>

$\mathrm{C}_{15} \mathrm{H}_{16} \mathrm{O}_{3}$

$\mathrm{M}=244,29$

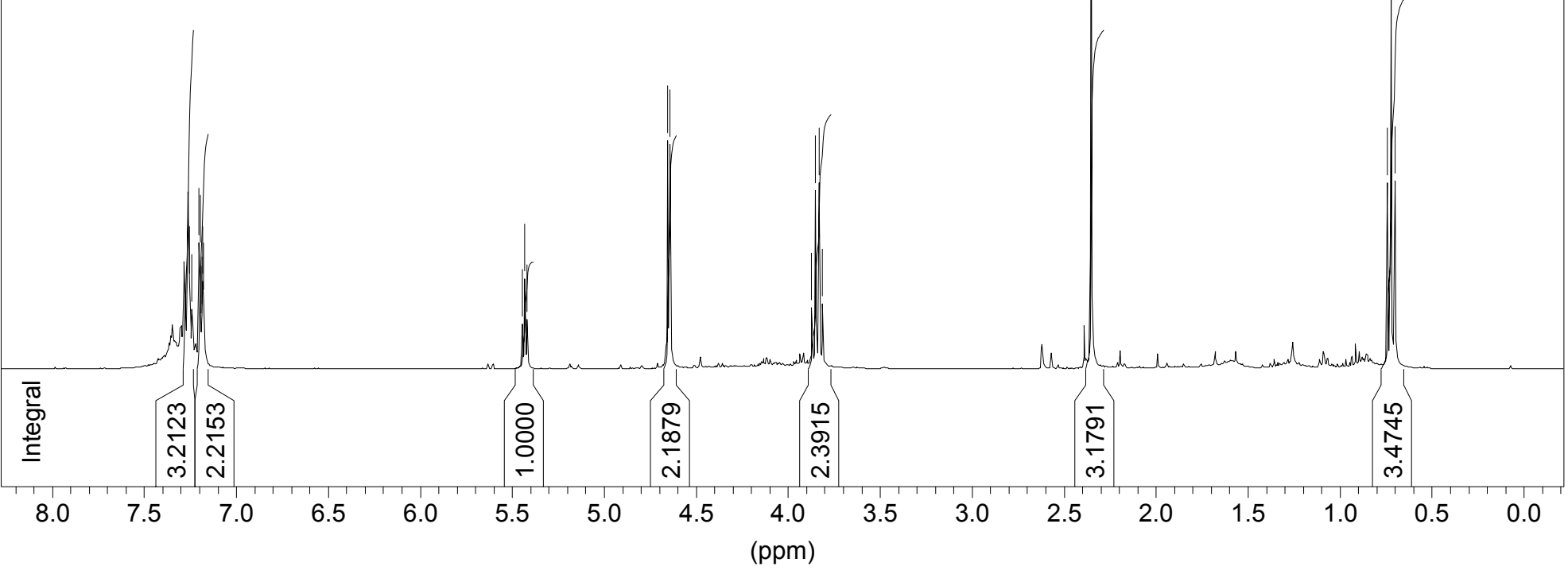

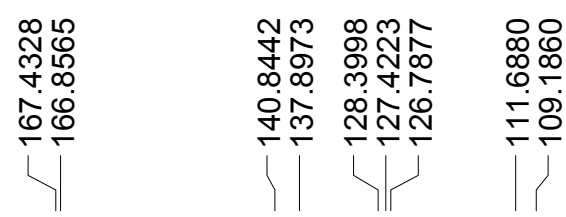

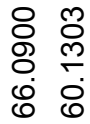

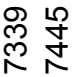

$\stackrel{\infty}{\circ} \stackrel{\dot{m}}{\stackrel{1}{m}}$

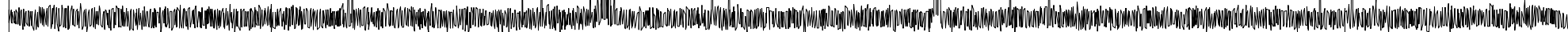




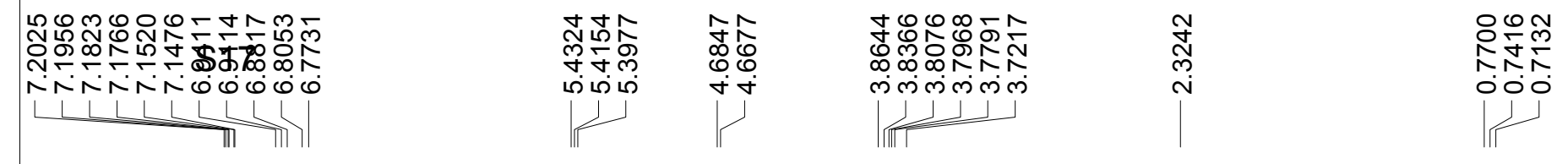

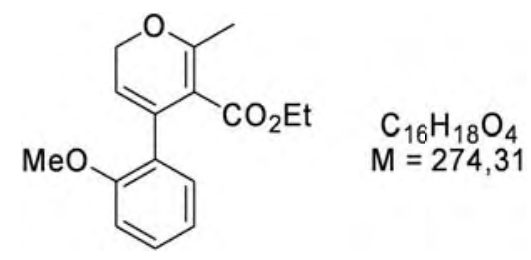
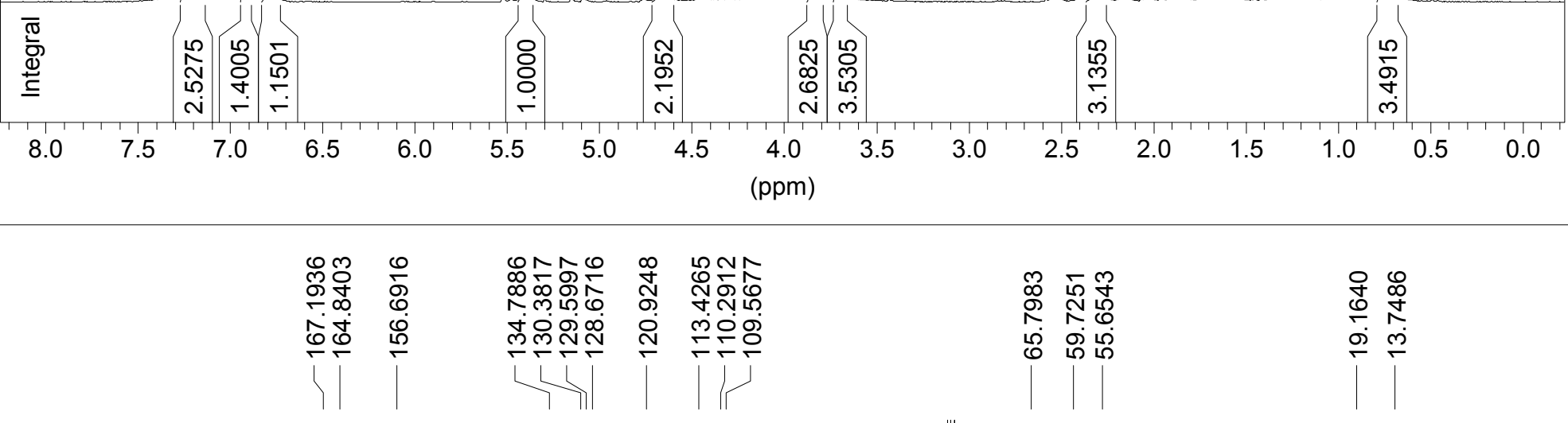


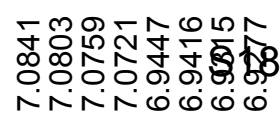

NNN

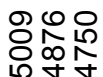

เก

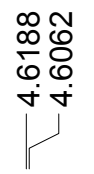

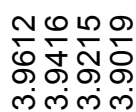

$\stackrel{n}{n}$

象守

$\mathrm{C}_{13} \mathrm{H}_{14} \mathrm{O}_{3} \mathrm{~S}$

$M=250,31$<smiles>CCOC1=C(C)OCC=C1c1ccsc1</smiles>
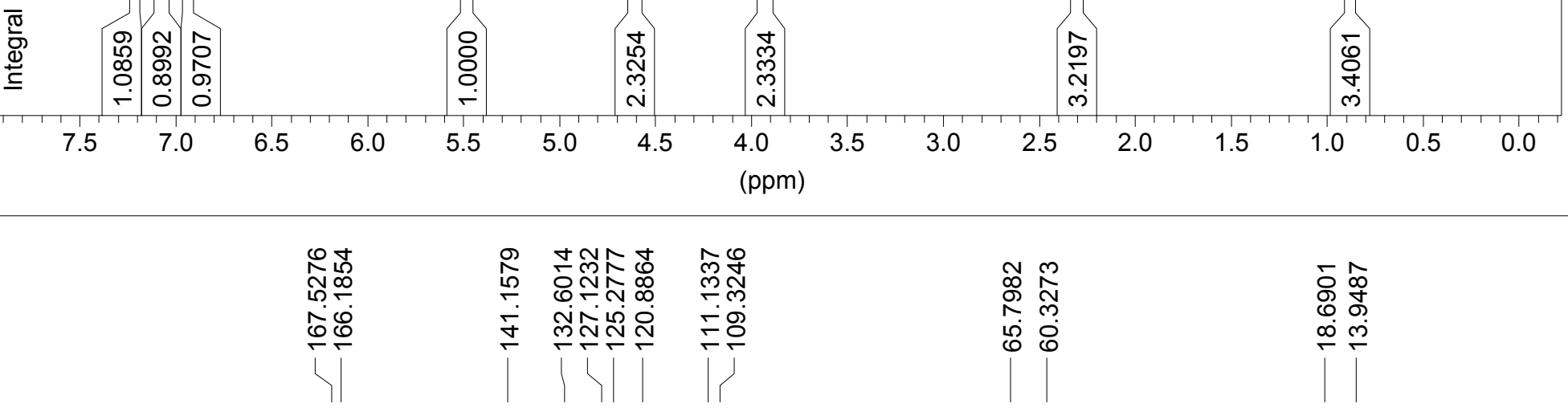

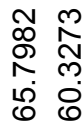

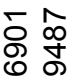




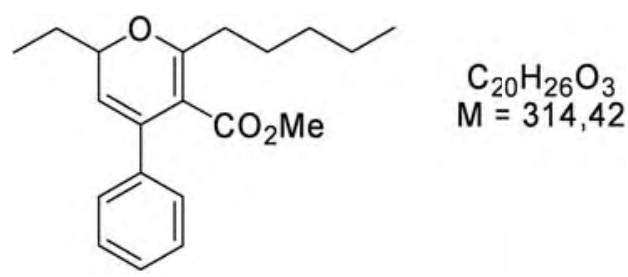

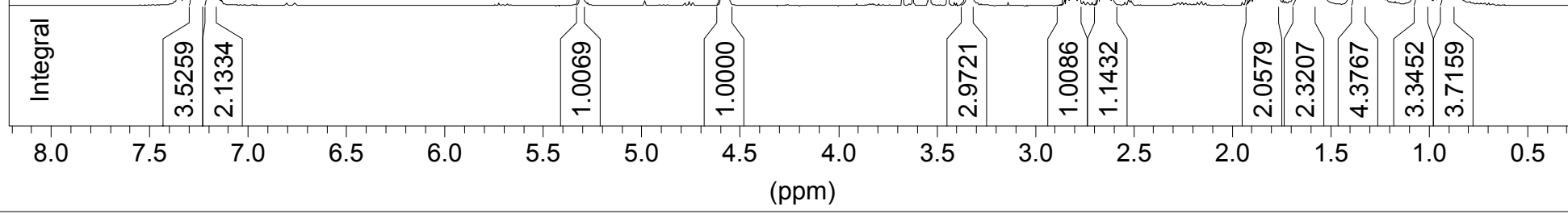

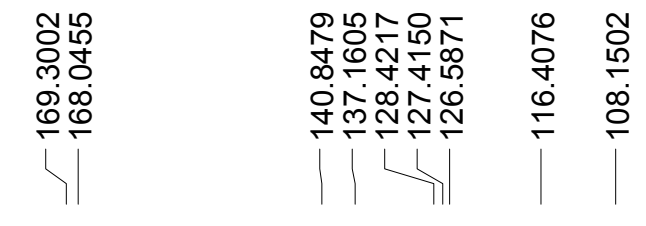
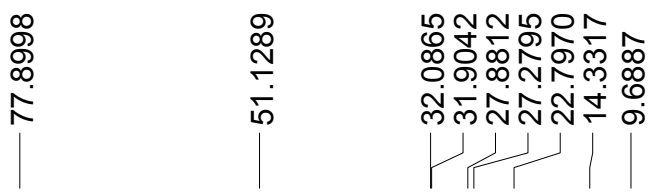

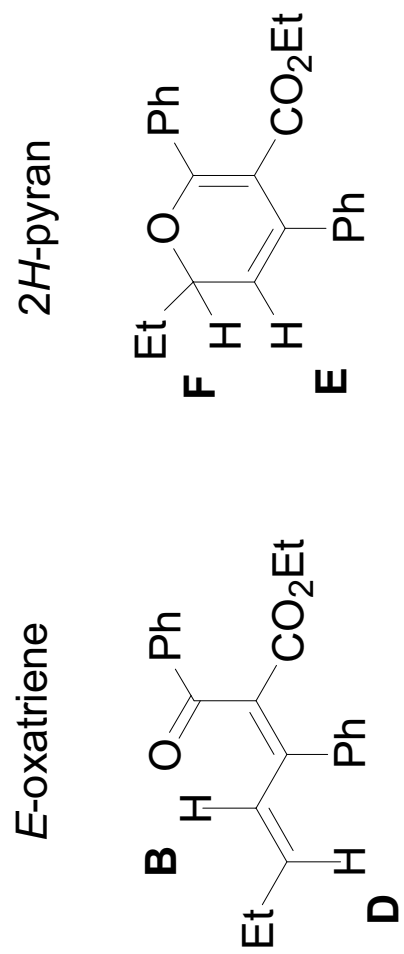

†882 9

$90 \varepsilon \varepsilon^{\prime} 9$

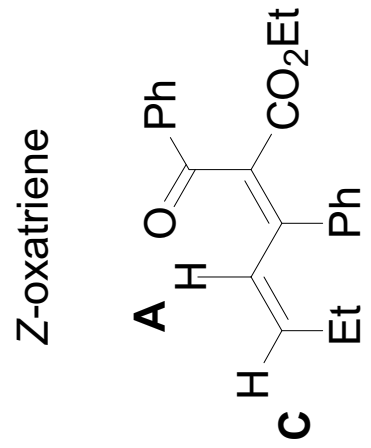

\section{$\boldsymbol{m}$}

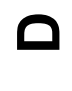

0

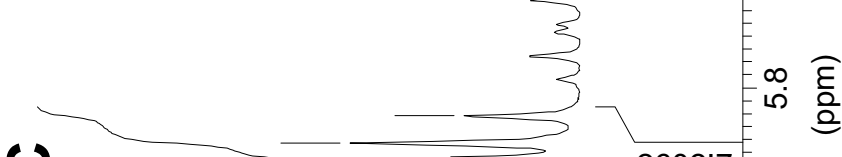


$\mathrm{O}$ $\begin{array}{cc} & \mathrm{C}_{22} \mathrm{H}_{28} \mathrm{O}_{3} \\ \mathrm{CO}_{2} \mathrm{Et} & \mathrm{M}=340,46\end{array}$

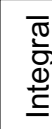

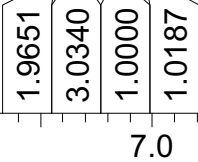

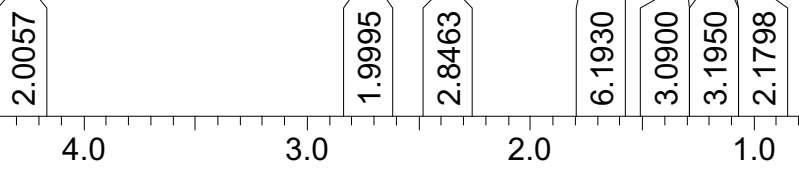

(ppm)

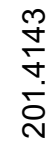

$\stackrel{+}{\circ}$
$\stackrel{0}{0}$
0
0

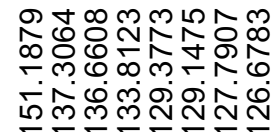

$\underset{\substack{N\\}}{\stackrel{0}{0}}$

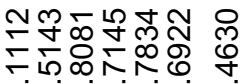

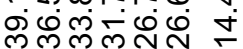

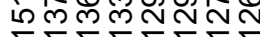

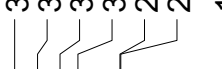




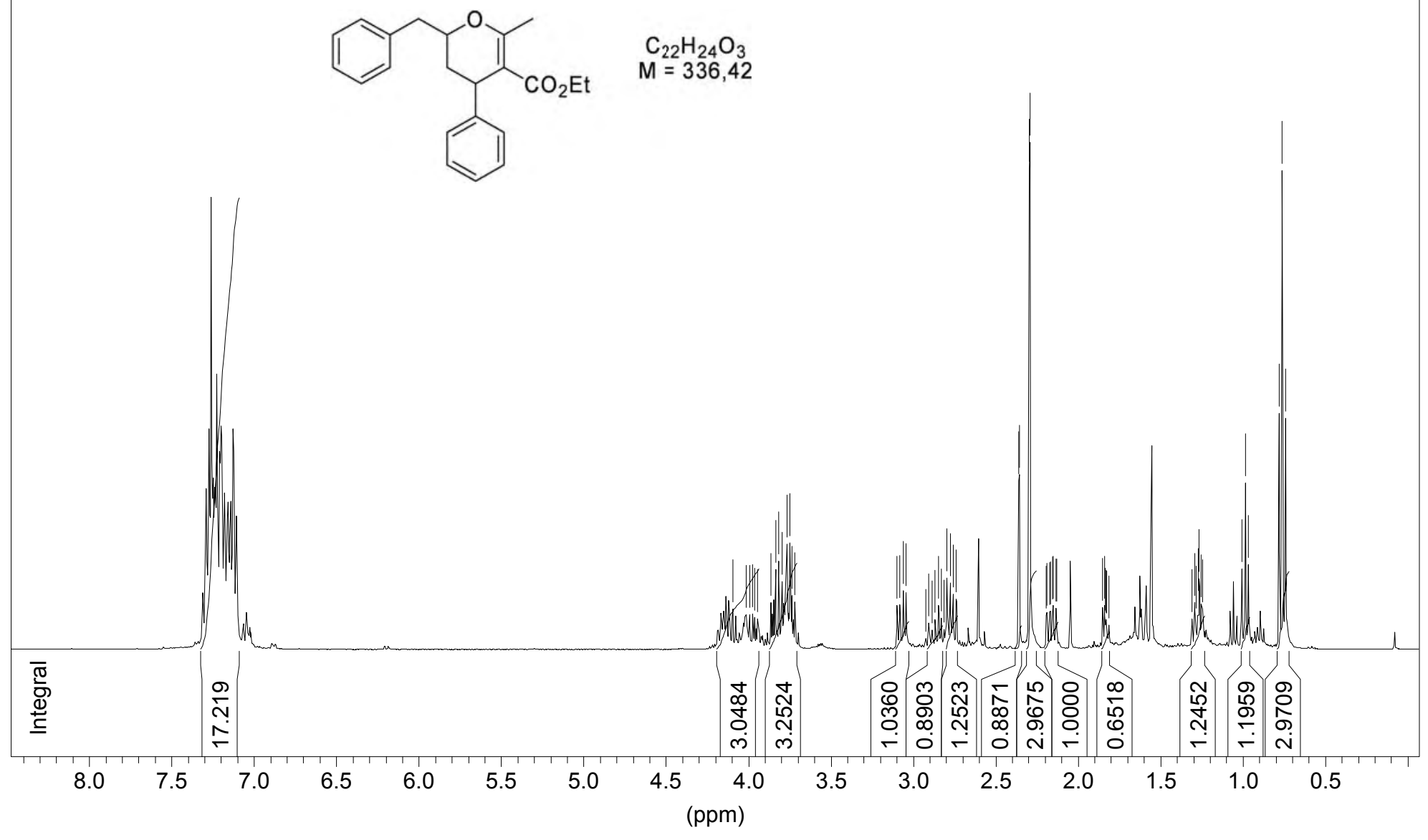

น้ำ

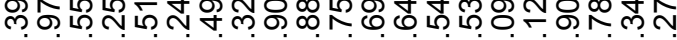

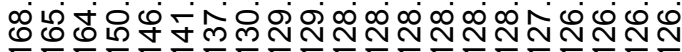
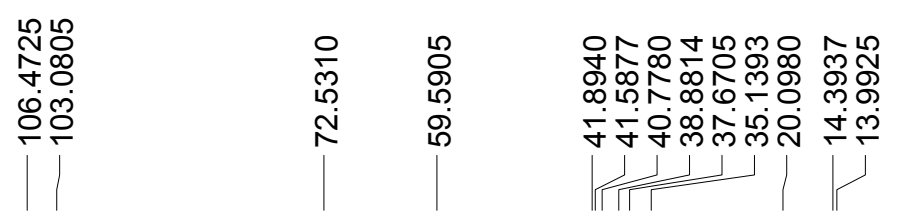
$\mathrm{S} 23$

అִำ

กิ๊

NiñNin

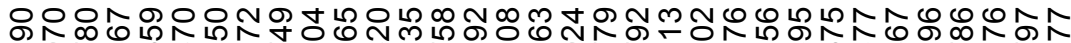

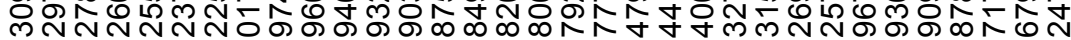

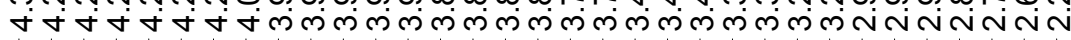

ธุำ

œळ .0ं

$\mathrm{C}_{22} \mathrm{H}_{24} \mathrm{O}_{5}$ $M=368,42$<smiles>CCOC(=O)C1=C(C)O[C@@H](Cc2ccccc2)[C@H](O)[C@H]1O</smiles>

$\mathrm{HO} \mathrm{Ph}$
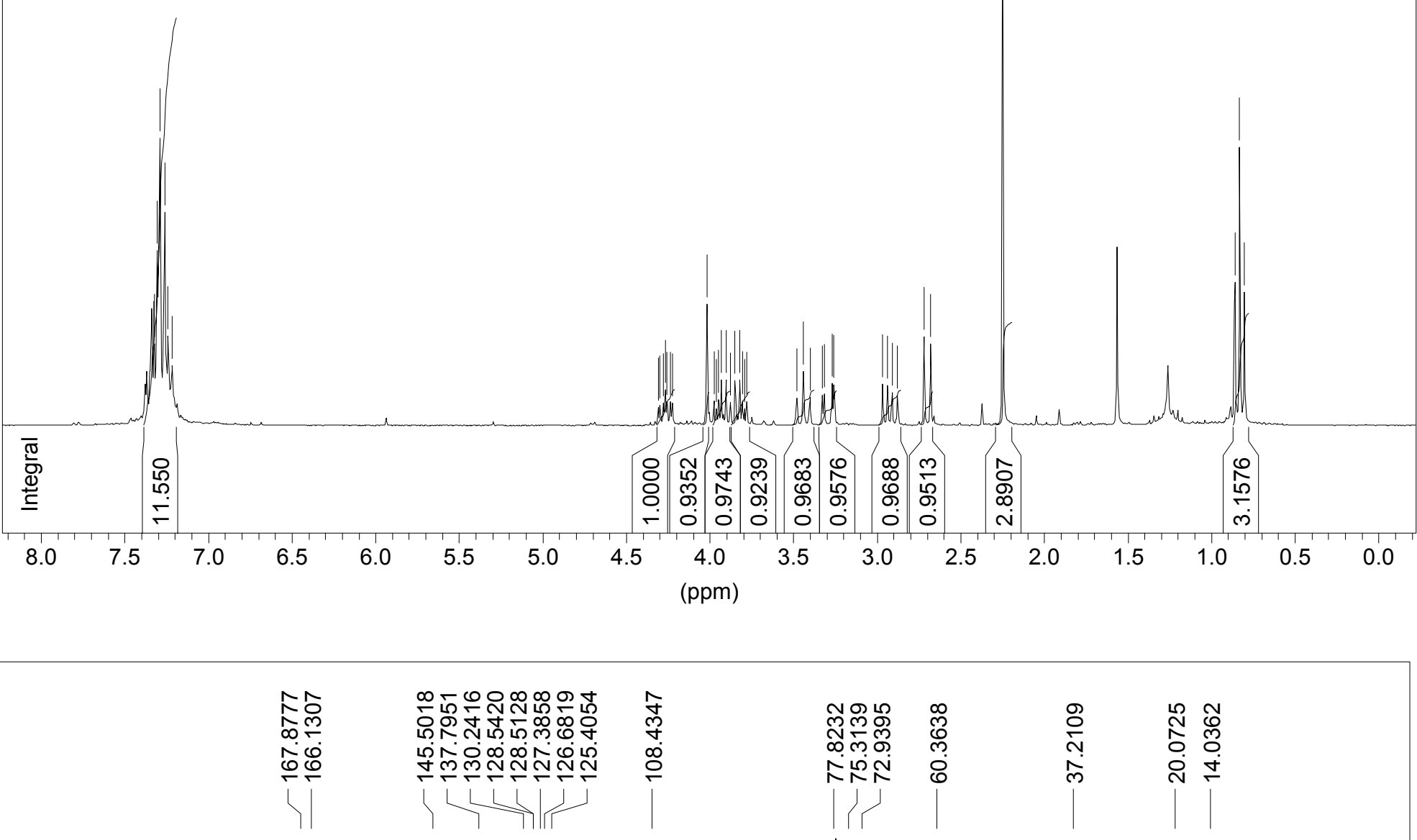

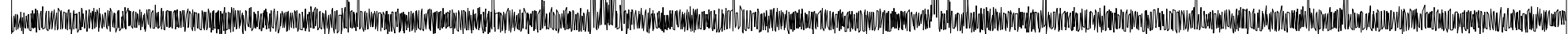




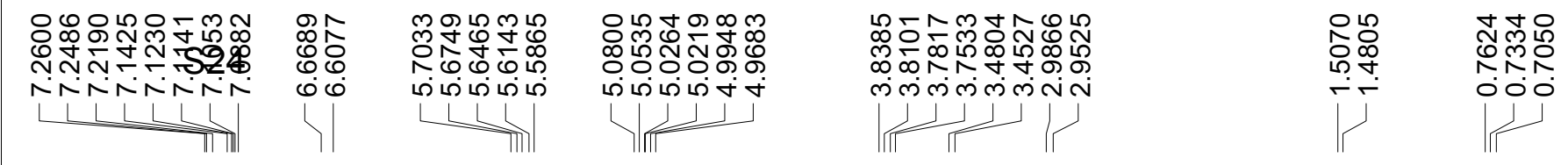<smiles>CCOC(=O)C(=CC=Cc1ccccc1)C(C)O</smiles>
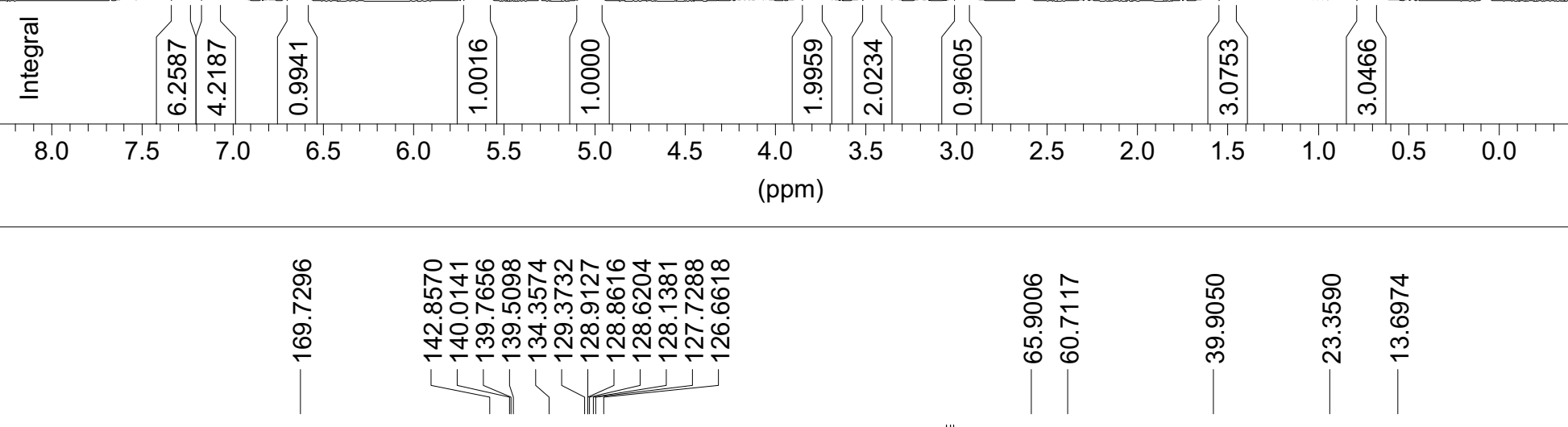